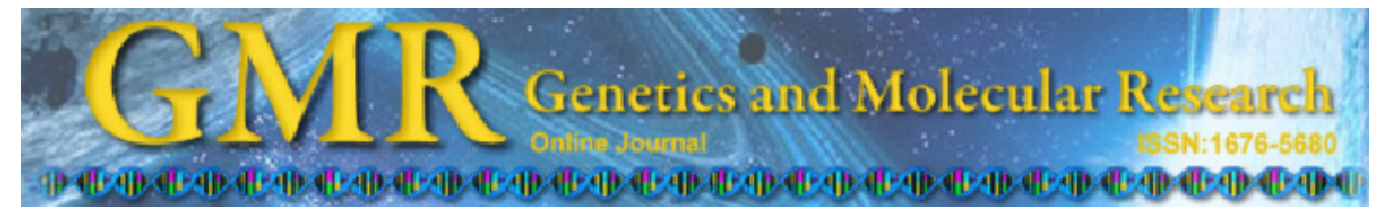

Short Communication

\title{
Utility of DNA barcoding for rapid and accurate assessment of bat diversity in Malaysia in the absence of formally described species
}

\author{
J.-J. Wilson ${ }^{1,2 *}$, K.-W. Sing ${ }^{1 *}$, M.R.A. Halim², R. Ramli ${ }^{2}$, \\ R. Hashim ${ }^{2}$ and M. Sofian-Azirun ${ }^{2}$ \\ ${ }^{1}$ Museum of Zoology, Faculty of Science, University of Malaya, \\ Kuala Lumpur, Malaysia \\ ${ }^{2}$ Institute of Biological Sciences, Faculty of Science, \\ University of Malaya, Kuala Lumpur, Malaysia \\ *These authors contributed equally to this study. \\ Corresponding author: J.-J. Wilson \\ E-mail: johnwilson@um.edu.my
}

Genet. Mol. Res. 13 (1): 920-925 (2014)

Received November 30, 2012

Accepted June 29, 2013

Published February 19, 2014

DOI http://dx.doi.org/10.4238/2014.February.19.2

\begin{abstract}
Bats are importantflagship species for biodiversity research; however, diversity in Southeast Asia is considerably underestimated in the current checklists and field guides. Incorporation of DNA barcoding into surveys has revealed numerous species-level taxa overlooked by conventional methods. Inclusion of these taxa in inventories provides a more informative record of diversity, but is problematic as these species lack formal description. We investigated how frequently documented, but undescribed, bat taxa are encountered in Peninsular Malaysia. We discuss whether a barcode library provides a means of recognizing and recording these taxa across biodiversity inventories. Tissue was sampled from bats trapped at Pasir Raja, Dungun Terengganu, Peninsular Malaysia. The DNA was extracted and the COI barcode region amplified and sequenced. We identified 9 species-level taxa within our samples, based on analysis of the DNA barcodes. Six specimens matched to four previously
\end{abstract}


documented taxa considered candidate species but currently lacking formal taxonomic status. This study confirms the high diversity of bats within Peninsular Malaysia (9 species in 13 samples) and demonstrates how DNA barcoding allows for inventory and documentation of known taxa lacking formal taxonomic status.

Key words: DNA barcoding; Bats; Biodiversity; Malaysia; Species identification; Hipposideros

\section{INTRODUCTION}

Bats (Order Chiroptera) play a crucial role in nature conservation. Action to protect bat species, and specifically their habitat, confers protection to a broad range of taxa that are also restricted or endemic to the natural area under threat. The assessment of bat species diversity (alpha, beta) is important for the designation of new protected areas and for directing and prioritizing conservation measures within existing ones. However, despite being well-studied and taxonomically "well-known", diversity in Southeast Asia is considerably underestimated in the common checklists and in field guides.

The incorporation of DNA barcoding (Ratnasingham and Hebert, 2007) into bat surveys has suggested the frequent occurrence of cryptic taxa overlooked by traditional (morphological) methods (Borisenko et al., 2008; Francis et al., 2010). The recognition of these cryptic taxa provides a more informative record of diversity; however, this is problematic, as they lack formal taxonomic status (i.e., formal description). They are so-called "dark" taxa (Maddison et al., 2012).

Here, we investigated how often dark bat taxa are encountered in Peninsular Malaysia and discuss how the barcode library provides a means of recognizing and recording these taxa across biodiversity inventories in the absence of formal taxonomic names.

\section{MATERIAL AND METHODS}

Bats were trapped at Pasir Raja, Dungun Terengganu, Malaysia $\left(4^{\circ} 35.413^{\prime} \mathrm{N}\right.$, $102^{\circ} 57.097^{\prime} \mathrm{E}$ ) with permission from the Department of Wildlife and National Parks (Perhili$\tan )$ to RR, using harp-traps, during routine fieldwork conducted by the Institute of Biological Sciences, University of Malaya. Tissue was sampled from wing membranes into $99 \%$ ethanol using standard protocols (AMNH, 2012).

DNA was extracted from 13 specimens using a Nucleospin kit (Machery-Nagel, Germany) following manufacturer instructions. The DNA barcode segment of COI mtDNA ( $\sim 650$ bp) was PCR amplified using standard techniques (Wilson, 2012) and the primer pair C_VF1LF and C_V1LR (Ivanova et al., 2012). PCR products were sent for sequencing at a commercially available service (MYTACG-Kuala Lumpur, Malaysia), using the M13R tail primer (Ivanova et al., 2012).

The resulting DNA barcodes were uploaded to the BOLD (Ratnasingham and Hebert, 2007) project "Small Mammals Malaysia2" and assigned to species on the basis of matches to the "Full Database" (Table 1). A neighbor-joining tree of K2P distances was plotted in MEGA 5 (Tamura et al., 2011) using all the publicly available Hipposideros barcodes from BOLD (Ratnasingham and Hebert, 2007). 


\section{RESULTS}

DNA barcodes were successfully amplified and sequenced from 13 specimens. On the basis of the DNA barcode results, we found that the sample contained 9 species (Table 1). Of these 9 species, 4 species (44\%) were dark taxa within the genus Hipposideros (Figure 1).

\begin{tabular}{|c|c|c|c|}
\hline Field ID & Name of the closest match & $\begin{array}{c}\text { Similarity with closest } \\
\text { match }(\%)\end{array}$ & $\begin{array}{c}\text { GenBank accession No. of } \\
\text { closest match }\end{array}$ \\
\hline b2 & Phoniscus atrox & 99.82 & HM541211 \\
\hline b3 & Hipposideros cervinus & 100 & HM540358 \\
\hline b4 & Rhinolophus lepidus & 98.55 & HM541573 \\
\hline b9 & Rhinolophus affinis & 100 & HM541414 \\
\hline b14 & Rhinolophus lepidus & 99.81 & HM541573 \\
\hline b21 & Hipposideros cervinus & 100 & HM540358 \\
\hline b30 & Hipposideros bicolor 31 & 99.64 & HM540344 \\
\hline b56 & Phoniscus atrox & 99.82 & HM541211 \\
\hline b60 & Hipposideros cf. bicolor & 99.82 & HM540379 \\
\hline b67 & Rhinolophus affinis & 98.71 & HM541414 \\
\hline b69 & Hipposideros ef. larvatus & 100 & HM240403 \\
\hline b74 & Murina aenea & 99.64 & HM540928 \\
\hline b91 & Hipposideros cf. larvatus & 100 & HM540403 \\
\hline
\end{tabular}

\section{DISCUSSION}

Field identifications of bats in Southeast Asia can be extremely challenging (Medway, 1978) even though comprehensive field guides are available (e.g., Francis, 2001). Definitive identifications require analysis of internal morphology (skull, dentition) and often comparison with reference material (Borisenko et al., 2008; Francis et al., 2010). Field identifications remain questionable unless voucher specimens are retained which is often impossible due to government controls and ethical concerns. An alternative is to use a bat detector - a device that detects the presence of bats and attempts species determination based on echolocation ultrasound signals. However, echolocation call frequency is influenced by various factors: body size, body condition and humidity, as well as the presence of ecologically similar species, limiting its utility for species identification (Guillén et al., 2000).

DNA barcoding offers a means of overcoming these taxonomic impediments by enabling the identification of a specimen to a species by comparing a standardized fragment of its DNA against a library of DNA fragments of known origin. DNA can be recovered, routinely, from small tissue samples, non-lethally, for example, from hair and wing punches (Pfunder et al., 2004; AMNH, 2012). Molecular approaches have been applied to bat taxonomy in Southeast Asia and have highlighted the presence of many "cryptic" species (Francis et al., 2010). A combination of molecular and echolocation call analyses has validated a number of cryptic species within the family Hipposideridae (Kingston et al., 2001; Jones and Barlow, 2004; Thabah et al., 2006). While these taxa have been documented for over 10 years, their inclusion in checklists is problematic since they currently lack formal taxonomic status; they are "dark" taxa (Maddison et al., 2012).

In our study, we found that dark taxa are frequently encountered during routine bio- 


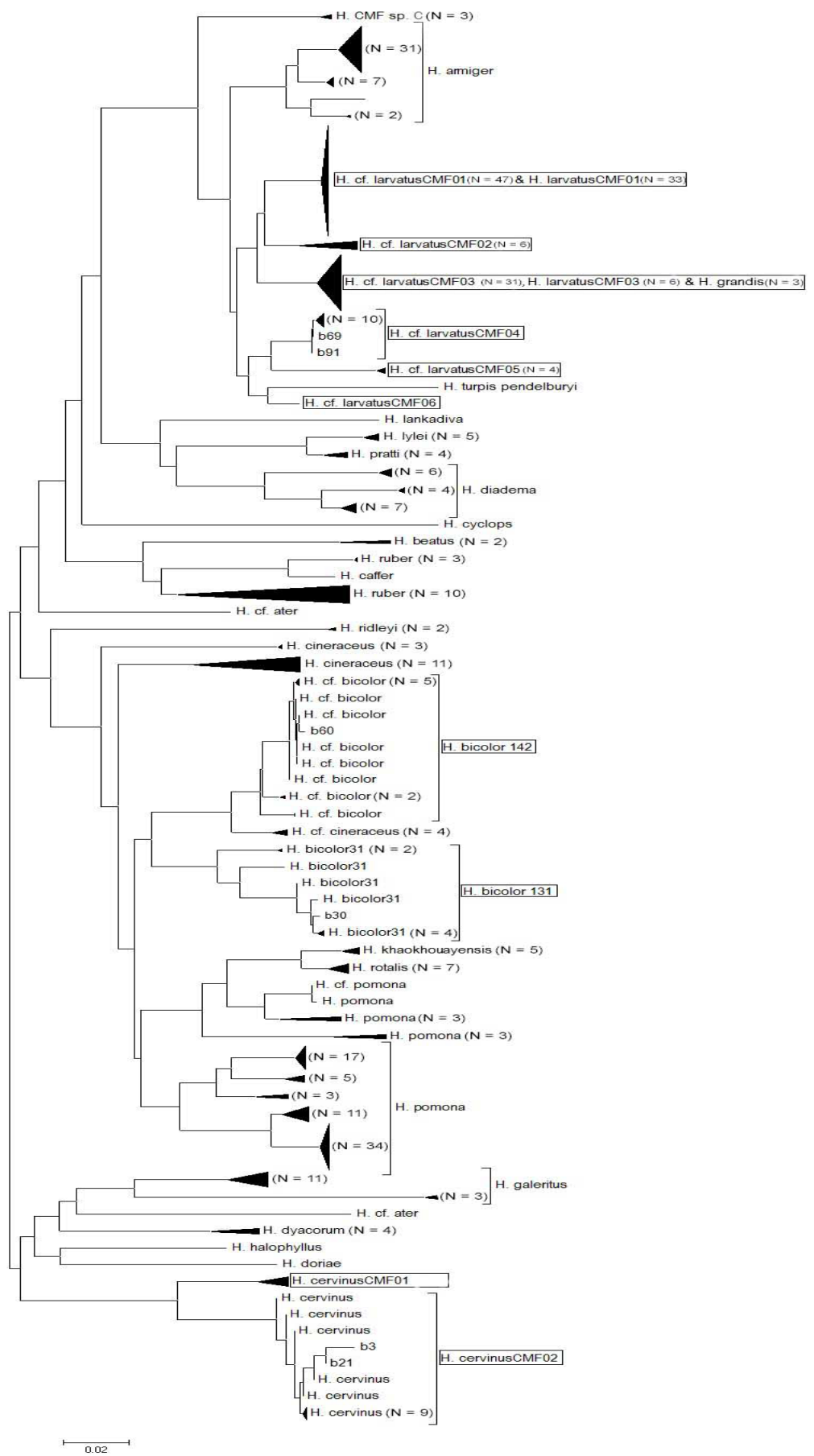

Figure 1. Neighbor-joining tree of K2P distances for all the publicly available Hipposideros DNA barcodes from BOLD. Some clades have been compressed to triangles due to the large number of individuals. 
diversity inventory of bats in Peninsular Malaysia. Our records of Hipposideros cf. larvatusCMF04, $H$. bicolor142, $H$. bicolor131 and $H$. cervinusCMF02 would not have been possible without the integration of DNA barcoding into our inventory.

We suggest that routine inclusion of DNA barcoding in the assessment of tropical bat diversity not only has the advantage of providing a means of accurate yet non-lethal species identification but also allows for the recognition and documentation of dark taxa across inventories providing a more precise and accurate estimate of alpha and beta diversity. Due to the well-recognised taxonomic impediment, the number of dark taxa is only going to increase (Maddison et al., 2012), but the presence of these species cannot be ignored. DNA sequences are already the de facto universal communication tool, providing anchor points for data associated with specimens and (undescribed) species (Wilson, 2011). Due to a competitive market place and rapid technological advances, a DNA barcode can be obtained very easily and inexpensively for less than US\$ 10 per specimen in Malaysia, making the method easily accessible to biodiversity researchers.

\section{ACKNOWLEDGMENTS}

Research supported by the University of Malaya UMRG (\#RG158/12SUS to J.J. Wilson) and the University of Malaya Special Grant to the Museum of Zoology (\#A-21010DA322-B29000).

\section{REFERENCES}

AMNH - American Museum of Natural History (2012). Available at [http://research.amnh.org/vz/mammalogy/ donating-bat-tissue-and-hair-samples-genomic-and-stable-isotope-studies/wing-punch-and-hair-sampling] . Accessed November 23, 2012.

Borisenko AV, Lim BK, Ivanova NV, Hanner RH, et al. (2008). DNA barcoding in surveys of small mammal communities: a field study in Suriname. Mol. Ecol. Resour. 8: 471-479.

Francis CM (2001). Mammals of the South-East Asia. 1st edn. New Holland Publishers, London.

Francis CM, Borisenko AV, Ivanova NV, Eger JL, et al. (2010). The role of DNA barcodes in understanding and conservation of mammal diversity in southeast Asia. PLoS One 5: e12575.

Guillén A, Juste J and Ibáñez C (2000). Variation in the frequency of the echolocation calls of Hipposideros ruber in the Gulf of Guinea: an exploration of the adaptive meaning of the constant frequency value in rhinolophoid CF bats. $J$. Evol. Biol. 13: 70-80.

Ivanova NV, Clare EL and Borisenko AV (2012). DNA barcoding in mammals. Methods Mol. Biol. 858: 153-182.

Jones G and Barlow KE (2004). Echolocation in Bats and Dolphins. In: Cryptic species of Echolocating Bats (Thomas JA, Moss CF and Vater M, eds.). University of Chicago Press, Chicago, 349.

Kingston T, Lara MC, Jones G, Akbar Z, et al. (2001). Acoustic divergence in two cryptic Hipposideros species: a role for social selection? Proc. R. Soc. Lond. B Biol. Sci. 268: 1381-1386.

Maddison DR, Guralnick R, Hill A, Reysenbach AL, et al. (2012). Ramping up biodiversity discovery via online quantum contributions. Trends Ecol. Evol. 27: 72-77.

Medway L (1978). The Wild Mammals of Malaya (Peninsular Malaysia) and Singapore. 2nd edn. Oxford University Press, New York.

Pfunder M, Holzgang O and Frey JE (2004). Development of microarray-based diagnostics of voles and shrews for use in biodiversity monitoring studies, and evaluation of mitochondrial cytochrome oxidase I vs. cytochrome $b$ as genetic markers. Mol. Ecol. 13: 1277-1286.

Ratnasingham S and Hebert PD (2007). BOLD: The Barcode of Life Data System (http://www.barcodinglife.org). Mol. Ecol. Notes 7: 355-364.

Tamura K, Peterson D, Peterson N, Stecher G, et al. (2011). MEGA5: molecular evolutionary genetics analysis using maximum likelihood, evolutionary distance, and maximum parsimony methods. Mol. Biol. Evol. 28: 2731-2739. 
Thabah A, Rossiter SJ, Kingston T, Zhang S, et al. (2006). Genetic divergence and echolocation call frequency in cryptic species of Hipposideros larvatus s.l. (Chiroptera: Hipposideridae) from the Indo-Malayan region. Biol. J. Linn. Soc. Lond. 88: 119-130.

Wilson JJ (2011). Taxonomy and DNA sequence databases: A perfect match? Terr. Arthropod Rev. 4: 221-236.

Wilson JJ (2012). DNA barcodes for insects. Methods Mol. Biol. 858: 17-46. 